\title{
An Automatic Segmentation Method for MRI Multiparametric Volumes
}

\author{
Sonia Nardotto and Silvana G. Dellepiane
}

\begin{abstract}
Purpose of this work is the design and implementation of an automated method for digital volume segmentation, based on multi-parametric densities, fuzzy topology, and adaptive growth mechanism. The processing objective is the global segmentation of the digital volume, that is its partitioning into significant connected subsets, in a fully automatic way. The main advantage consists in the very nature of the algorithm that enables the automatic segmentation by running an iterative process that adapts to the volume at hand and does not require any user intervention. The designed method can be applied to multi-parametric volumes where different characteristics are available to analyze the same target. The robustness of the method has been evaluated and verified through statistical parameters, that will be discussed below, after application on volumes of biomedical images obtained through Magnetic Resonance Imaging.
\end{abstract}

Index Terms-Segmentation, fuzzy processing, connectedness, multi-parametric data fusion.

\section{INTRODUCTION}

The purpose of this work is the region partitioning of digital volumes using a method based on topological fuzzy membership values. This method shows various relevant features such as being totally automatic, because it does not require any user intervention, and it is adaptive to the actual data under analysis. It also proves to be independent from parameters and it does not make use of any predefined model. Furthermore, it performs data-fusion since it is able to integrate more volumes at the same time, in order to gain more information. This can be very useful in the field of medical image analysis because, for example for Magnetic Resonance Imaging, it allows an integrated exploitation of T1-weighted and T2-weighted volumes in diagnostic task.

As it is well known, on the contrary of Computed Tomography (CT), in Magnetic Resonance Imaging (MRI), a single homogeneous biological tissue is characterized by significant variability of the grey level intensities that represent a qualitative measure instead than a quantitative one. Such features cannot be numerically modeled, as it happens with the Hounsfield number of CT modality. At the same time, the contrast between various tissues is usually very smooth, especially because of the tomographic partial-volume effect.

Due to these aspects, one of the major problems in biomedical image processing and a subject of extensive research work, is the image segmentation, for a fast

Manuscript received July 24, 2013; revised October 3, 2013.

S. Nardotto is with Università degli Studi di Genova, DITEN, via Opera Pia 11a - I16145 Genova, Italy (e-mail: sonia.nardotto@edu.unige.it).

S. G. Dellepiane is with Università degli Studi di Genova, via Opera Pia 11a-I16145 Genova, Italy (e-mail: silvana.dellepiane@unige.it). identification of regions of interest (ROI), or for the global segmentation able to localize all the significant regions of the image (or volume). Specifically, the segmentation of MRI images and volumes is still an open research point. The final aim might be a subsequent processing, such as the extraction of measures from regions or the classification of the image or its portions thus recognizing a possible pathology.

Starting from the multi-seed approach proposed in [1] and [2], the aim of the proposed method is to develop an algorithm using more than one volume to take into account different features, for a better location of ROI and to extend this approach by using more seed points which are automatically selected with the goal of a global processing of the volume that gives as a result an unsupervised, automatic vectorial segmentation.

Since the basic algorithm is based on fuzzy membership evaluation, intermediate results are exploited for the combination of subsequent iterative steps.

The method starts from a random single seed point and ends with the complete segmentation of the image/volume automatically identifying other intermediate seed points. Each iterative step finds a new significant seed through the analysis of the intermediate fuzzy connectivity maps. When the stop condition is verified, a map of labels is generated from the final Total-Connectedness Map. The final result is the segmented volume, together with a list of significant seed points. Due to the complete independence from models and parameters, the method can also be used in application domains different from the medical field.

\section{RELATED WORKS}

The first proposed seed-segmentation method was the original work of Seeded-region-Growing (SRG) proposed by Adams and Bishof [3]. SGR is simple but gives good results, even if they are not optimal since dependent on the order of analysis, [4]. For this reason, some solutions have been suggested [4]-[6]. In all these works the classical probabilistic approach has been used and no fuzzy measure neither fuzzy processing is applied. The fuzzy framework, if used in the seed-growing approach, allows to relax the aggregation criterion by measuring the similarity of pixels to seed points, in terms of spatial relationship and also in terms of intensity similarity, without applying any test, avoiding any parameter and threshold setting and taking into account the inherent object material heterogeneity and imaging device artefacts.

Some methods of image segmentation, based on fuzzy concept are reported in the literature [7]-[9]; in addition to fuzzy clustering [9], fuzzy rule-based methods [7], [10]-[12], fuzzy thresholding [8], [13]-[15], fuzzy Markov Random Fields [16]-[18], and fuzzy region growing [19], [20] have 
been reported. Fuzzy connectedness can be defined in in terms of "intensity connectedness" [21] or in terms of "affinity" [22]. They take into account both feature space information and contextual-spatial relationships among pixels. (as opposite to fuzzy clustering that does not take into account any spatial dependency among pixels). The "relative fuzzy connectedness" has been proposed in [23] by Udupa et al., explaining how various objects in the image/volume "are let to compete among themselves in having spels as their members". In the experiments there proposed, multiple seeds were utilized to specify a class of objects and different co-objects in the background. A distinction between seeds corresponding to the class of interest and the seeds corresponding to the background are manually located by the user. Only one class at a time is segmented. Another work by Udupa is a vectorial image segmentation [24] whose results will be compared to the ones achieved by the present method.

The innovative method proposed in this paper is based on the fully automatic seed selection that is driven by intermediate connectivity results since this information is used to establish the position of new seed point. This operation is repeated iteratively until all the volume is segmented into meaningful regions.

Multiple sclerosis (MS) is a common chronic disease that predominantly involves the white matter of the central nervous system and often causes serious neurological disorders [25]. Unfortunately, the poor sensitivity of the standard tests currently used, does not permit objective and accurate quantification of the disease severity or of its change on its own or as a response to drug therapy. Magnetic resonance (MR) imaging has proven to be a very sensitive marker of the MS disease [26]. In fact macroscopic areas of damage or loss of myelin can be imaged with hyper or hypointensity with respect to the surrounding tissues.

The proposed method has been evaluated and compared in the application of MS plaques segmentation.

\section{PROPOSED METHOD}

This method is divided into five steps described in sections from $A$ to 3.5 :

\section{A. Fuzzy $\chi$-Connectedness Map}

The first seed point is here randomly placed, unlike the MSMC method [1], where seeds are manually selected by the user. Following [2], let $V$ be a generic 3D square lattice, where $v_{i} \in V$ with $i=1 \ldots,|V|$, is the $i$-th voxel. The intensity map $M$ is a function from $V$ to the scalar domain, representing the random field of the grey levels of an original digital volume. Given an appropriate rationale number, $r$, a fuzzy map $H$ is derived from $M$ where $\eta_{\left(v_{i}\right)}=r \cdot M\left(v_{i}\right)$ such that $\eta_{\left(v_{i}\right)}$ is a fuzzy value within the unit real interval $[0,1]$. Following paper [21], as a preliminary step, when a seed point is chosen, named $v_{a}$ the function $\chi^{v_{a}}$ from $\mathrm{V}$ to the scalar fuzzy domain is defined as

$$
\forall v_{i} \subset V \chi_{v_{i}}^{v_{a}}=1-\left|\eta_{\left(v_{i}\right)}-\eta_{\left(v_{a}\right)}\right|
$$

which gives a fuzzy value to each voxel according to the intensity similarity with the seed voxel. From [1], the fuzzy-intensity connectedness (or $\chi$-connectedness) map,

$$
C_{\chi^{v a}} \text {, is defined as }
$$

$$
\forall v_{i} \subset V C_{\chi^{v_{a}}}=\max _{P\left(\mathrm{v}_{a}, \mathrm{v}_{i}\right)}\left[\min _{z \in P\left(\mathrm{v}_{a}, \mathrm{v}_{i}\right)} \chi^{v_{a}}(\mathrm{z})\right]
$$

where $P\left(v_{a}, v_{i}\right)$ is a path, i.e., a connected sequence of voxels from $v_{a}$ to $v_{i}$.

Following the method described in [27] and [21], the above connectedness map is generated for the first seed point and all the subsequent ones.

\section{B. $\chi$-Vectorial Computation}

In medical imaging, it is sometimes necessary to evaluate multiple images, for example, in MRI the T1 volume and T2 volume are simultaneously evaluated, in order to establish or exclude the presence of a disease. For this reason, in this paper a vectorial fuzzy seed segmentation is proposed, unlike the method proposed in [2], where the volumes are taken one at a time.

The formulation of the $\chi$ field $\chi^{v_{a}}$ previously described in equation (1) is unchanged, then for each seed the respective $\chi_{j}{ }^{v_{a}}$, are calculated where $v_{a}$ indicates the seed point, and $j$ indicates the number of the volume.

Given a generic seed position, the two volumes are separately processed and the two $\chi$-fields are calculated. Subsequently they are integrated, by using the following formula, related with the Euclidean norm:

$$
\chi t=\sqrt{\chi_{1}^{2}+\chi_{2}^{2}}
$$

where $\chi_{1}$ is the $\chi$-map derived from the first volume and $\chi_{2}$ is the $\chi$-map of the second volume.

Therefore, only one connectivity map is derived from one seed, having exploited a two-parametric input information and the $C_{\chi^{t}}$ is generated by applying equation (2).

\section{Total Connectedness Map}

Like in paper [2], connectivity information independently derived from each generic seed $\mathrm{t}$, and contained in the related membership maps $C_{\chi^{t}}$, are merged into a single “Total-Connectedness Map" through a process of fuzzy union:

$$
C_{T}=\bigcup_{t=1}^{T} C_{\chi} t
$$

\section{Minimum Research and New Seed}

A novel automatic method for searching seeds is here proposed. The method in paper [2] proposes a random selection of a few seeds at each iterative step, followed by a procedure devoted to find and remove eventual redundant seeds.

With the aim of simplicity and faster computation, the new implemented method avoids redundant seeds thanks to a new search mechanism where only one seed point is added at each 
step.

Given the map of total connectivity, the new approach looks for the minimum value $\left(C_{\min }\right)$ and its location $\left(v_{p}\right)$ :

$$
\begin{gathered}
\forall v_{p} \in C_{T}\left(v_{P}\right) \\
C_{\text {min }}=\min \left[C_{T}\left(v_{p}\right)\right]
\end{gathered}
$$

Such a step has a two-fold reason. It is able to locate the so-called "residual voxels" which are not sufficiently well represented by the already selected seeds.

It also turns useful for the stop condition criterion as explained in the following subsection. The coordinates $(x, y$, $z$ ) of the minimum voxel from equation (5) are stored as new location where to place a seed for the subsequent calculation of the new map, which will be merged with the previous ones through equation (4).

\section{E. Stopping Criterion and Labeling}

Since connectivity looks for connected zones, when all zones have been segmented as separate regions, the connectivity can no longer increase. If we plot the minimum value at each processing step we typically obtain a graph similar to the one, shown in Fig. 1.

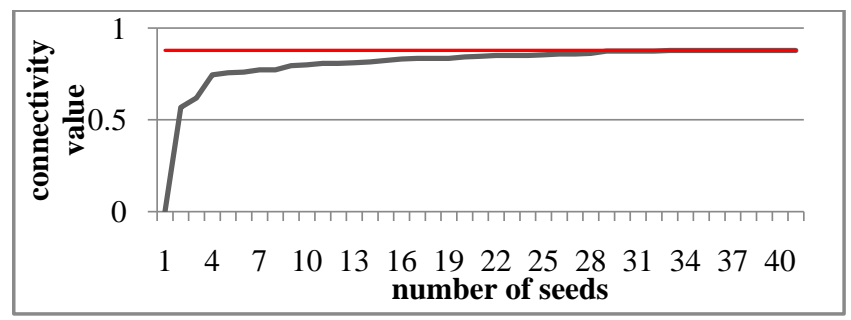

Fig. 1. Minimum connectivity value as a function of the seeds number.

When the graph reaches a plateau, it means that the data has been completely segmented and the stop condition is verified

One can then proceed with the final labeling, by creating the labeling map $\Lambda$ where each region is associated with a different label value.

In order to evaluate the method performances, MRI brain volumes have been considered, addressing the segmentation of intracranial brain tissues, i.e., Cerebrospinal Fluid (CSF), White Matter (WM) and Gray Matter (GM).

\section{RESULTS}

To make the test results comparable with other works in the literature and to achieve a quantitative numerical evaluation, we have used the Brainweb [28] dataset, which has complete volume phantoms available, that represent the correct segmentation result. The used volume size is $181 \times 217 \times 181$ voxels, spatial resolution being $1 \mathrm{~mm}^{3}$. Fig. 2 shows, as an example, the 100th brain slice of a considered volume affected by Multiple Sclerosis (MS) disease. The method generates the final-intensity-connectedness-map $C_{T}$ , derived from the previous ones through the fuzzy union process, and, more importantly, the label map 1 . As an example, the connectivity map generated from the combination of $\chi_{1}$ and $\chi_{2}$ maps is shown in Fig. 3, as dealing with the first seed point placed in a CSF area.

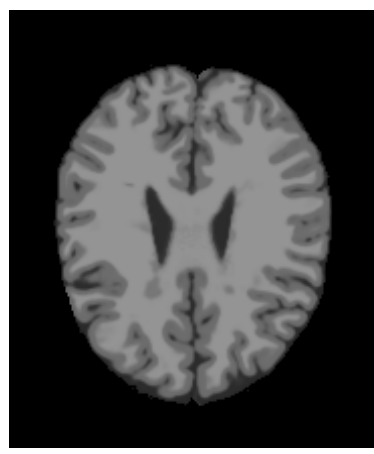

(a)

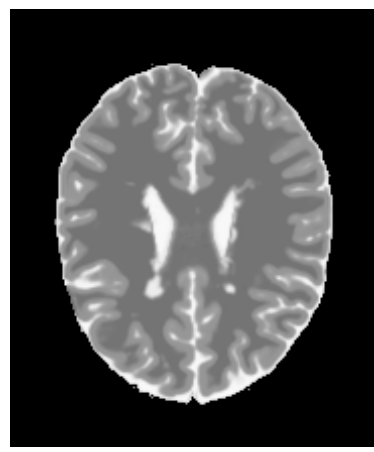

(b)
Fig. 2 (a). Simulated T1-weighted pathological MR image, 100th slice: 0\% noise. (b) Simulated T2-weighted MR image, 100th slice: $0 \%$ noise.

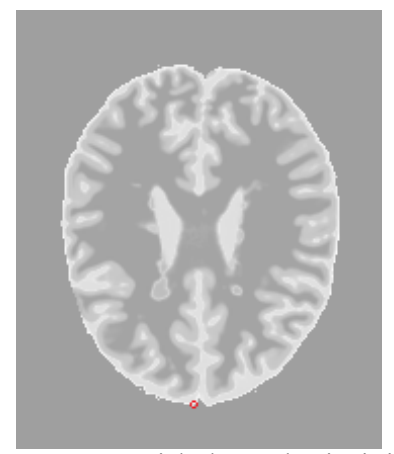

Fig. 3. $\chi$ vectorial. The seed point is in red circle

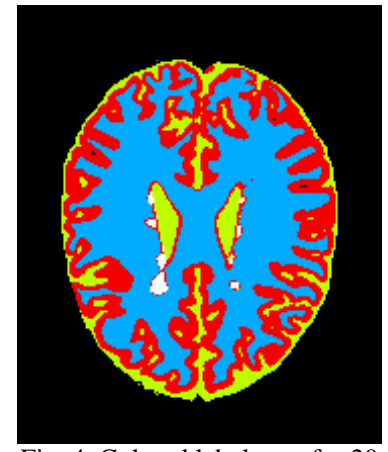

Fig. 4. Colored label map for 20 seed points.
The first research of connectivity minimum is carried on this map. Fig. 4 shows the related final label map $\Lambda$ where each label value is associated to a color for visualization purpose.

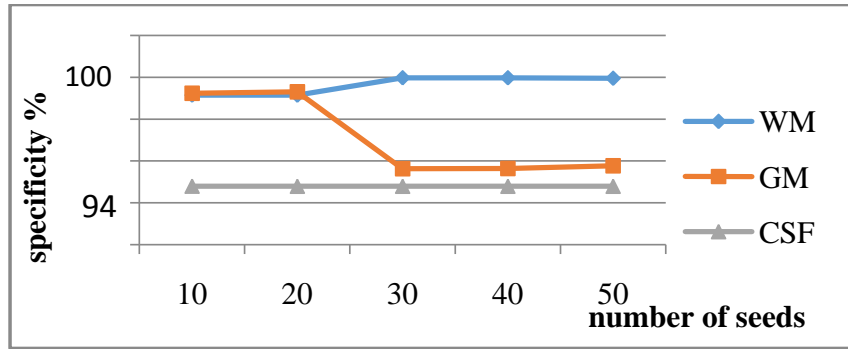

Fig. 5. Trend of specificity for WM, GM and CSF.

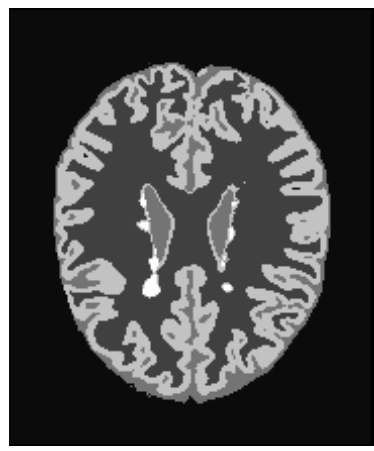

(a)

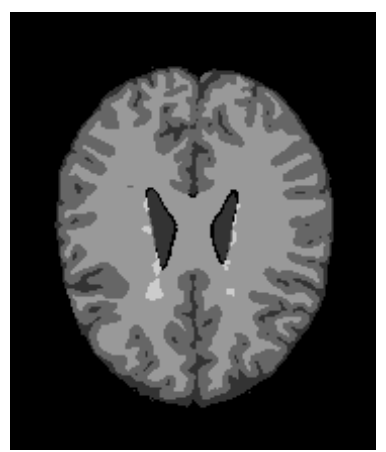

(b)
Fig. 6. Simulated pathological MR image, 100th slice: $0 \%$ noise (a) Label map for 20 seeds point; (b) Phantom.

A statistical evaluation of performances is based on the computation of parameters such as Accuracy, Sensitivity and Specificity [7]. The achieved results are compared to the true segmentation provided by the Brainweb phantom, by 
changing the final seeds number. As shown in Fig. 5 the Specificity value grows until about 30-40 seeds point for WM and CSF, but it decreases for GM. When this empirical evidence is used as a stop condition, a number equal to 20 seeds is chosen as a good compromise. The final result is show in Fig. 6 (left part) as compared with the phantom (right part). In Table I the obtained results in terms of sensitivity, specificity, accuracy, for $0 \% \mathrm{RF}, 0 \%$ noise; 20 seeds are reported.

TABLE I: ACCURACY RESULTS FOR PATHOLOGICAL MRI VOLUME, 0\% RF, $0 \%$ NOISE; 20 SEEDS

\begin{tabular}{llcc}
\hline \hline Class & Specificity & Sensitivity & Accuracy \\
\hline WM & $98.6874 \%$ & $97.5388 \%$ & $98.4684 \%$ \\
GM & $97.8387 \%$ & $96.9148 \%$ & $97.4108 \%$ \\
CSF & $99.4296 \%$ & $98.0334 \%$ & $98.9489 \%$ \\
MS & $99.9845 \%$ & $79.7836 \%$ & $99.9482 \%$ \\
\hline \multicolumn{2}{c}{ Overall Accuracy } & \multicolumn{2}{c}{$97.3881 \%$} \\
\hline \hline
\end{tabular}

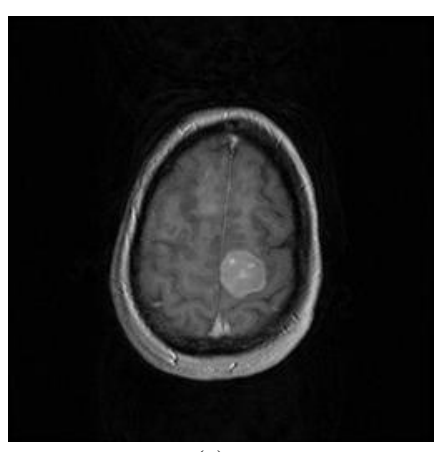

(a)

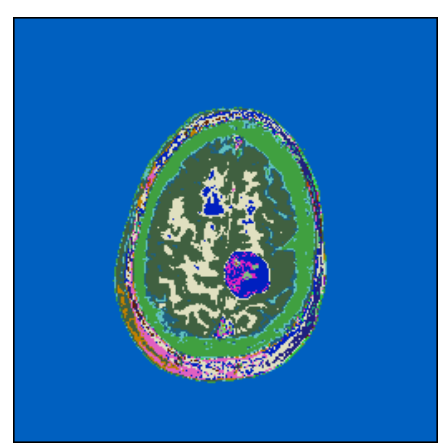

(b)
Fig. 7 (a). Real axial T1-weighted pathological MR image, 0\% noise.(b) Color label map for 40 seeds point

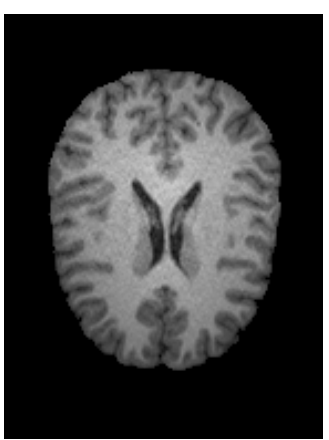

(a)

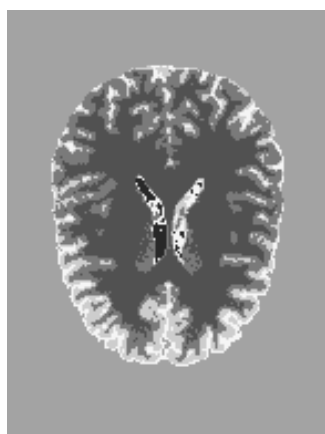

(b)

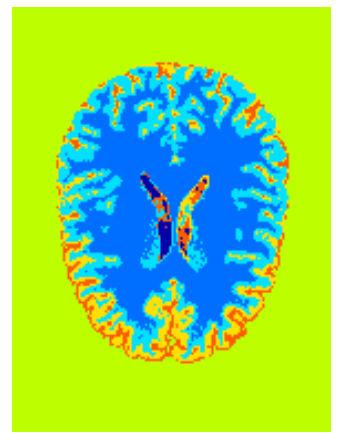

(c)

Fig. 8 (a). Real axial T1-weighted pathological MR image; (b) Label map of MR image; (c) LUT of label map.

A very good specificity, sensitivity and accuracy levels are achieved for MS class. In addition to the limited precision of the phantom one can notice that very often the segmentation error does not rely on the lesion identification but an under-segmentation of the single object occurs, so that the error is not in the detection of the lesion but in its size estimation. This may be less critical if the application goal is limited to guide the medical staff toward a quick identification of the position of the lesions, leaving to a further and deeper step the analysis of original volumes to understand the lesion situation.We performed a test on a real MR brain image in axial view (Fig. 7) provided by the Internet Brain Segmentation Repository (IBSR) [29] where the disease has been well recognized.

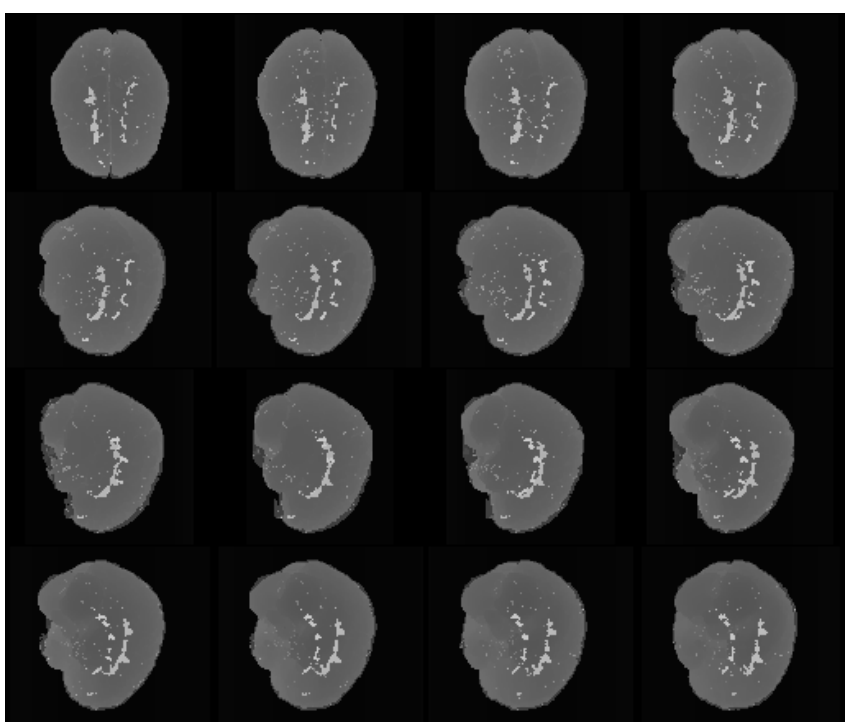

Fig. 9. MIP of multiple sclerosis lesion.
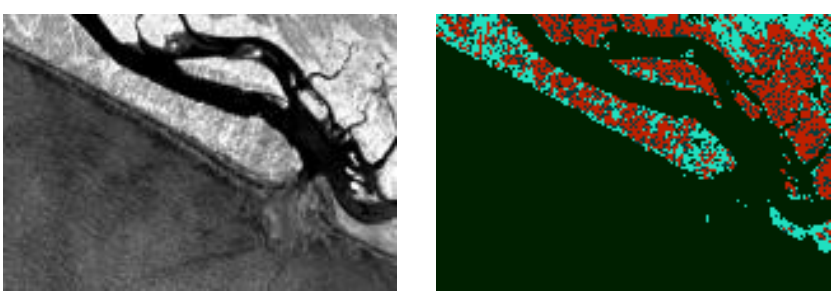

Fig. 10 (a). Satellite image; (b) Label map of satellite image.

In Fig. 8 we have a real T1- weighted MR brain image in axial view provided by Neuroimaging informatics tools and resources clearinghouse (NITRC) [30] and we can observe that all the most important regions in the image are correctly segmented.

Finally, we compared our method with another fuzzy vectorial method proposed by Udupa [24] and the results are shown in Table II. Fig. 9 shows a maximum intensity projection (MIP) of multiple sclerosis lesion, as a final result of our segmentation.

TABLE II: COMPARISON BETWEEN SPECIFICITY AND SENSITIVITY

PARAMETERS OBTAINED BY PROPOSED ALGORITHM AND METHOD PRESENTED IN [24] FOR VOLUMES WITH 0\% RF, $1 \%$ NOISE

\begin{tabular}{ccccc}
\hline \hline & \multicolumn{2}{c}{ Vectorial proposed } & \multicolumn{2}{c}{ Fuzzy vectorial [24] } \\
\hline \hline Class & Spec. & Sens. & Spec. & Sens. \\
\hline CSF & $99.14 \%$ & $98.14 \%$ & $93.90 \%$ & $94.10 \%$ \\
GM & $99.23 \%$ & $91.16 \%$ & $94.80 \%$ & $94.80 \%$ \\
WM & $94.80 \%$ & $99.83 \%$ & $96.80 \%$ & $96.40 \%$ \\
\hline \hline
\end{tabular}

We have performed a test on a satellite image, shown in Fig. 10. 


\section{CONCLUSION}

In conclusion, an automatic unsupervised fuzzy method for Magnetic Resonance image segmentation has been proposed. More precisely, the aim was a total segmentation of digital volumes without user intervention and this was performed with different phases that follow each other iteratively until the stop condition is verified. So starting from an existing method, new image processing steps were introduced: the automatic seed points selection, the new method for multi-parametric volumes integration, the three dimensional extension and a study for the stop condition.

An extensive evaluation session has been developed as dealing with robustness to the randomly selected initial seed, to the noise level, and to different case studies. In Section 4 quantitative performance evaluation of the method has been presented with special focus to the segmentation of intra-cranial tissues of simulated brain MRI volumes (normal and pathological).

As shown in the previous sections, good results have been obtained, comparable or superior to those presented in the literature but with some advantages. In fact, the present method does not make use of any supervised training phase for parameter estimation; no through a-priori information or models are used. Because this method is independent on the image content and on the image resolution, it works well for any kind of images.

Very interesting developments might be devised, addressing for instance, the integration of multi-modal and multi-temporal images, as well as their analysis as a support for location of changes.

\section{REFERENCES}

[1] S. G. Dellepiane, E. Angiati, and I. Minetti, "Multi-seed segmentation of tomographic volumes based on fuzzy connectedness," Advanced Intelligent Computing Theories and Applications. With Aspects of Artificial Intelligence, Springer, 2010, pp. 360-367.

[2] S. G. Dellepiane, V. Carbone, and S. Nardotto, "An automatic unsupervised fuzzy method for image segmentation,"Computational Modeling of Objects Presented in Images: Fundamentals, Methods and Applications, pp. 307, 2012.

[3] R. Adams and L. Bischof, "Seeded region growing," IEEE Trans. on Pattern Analysis and Machine Intelligence, vol. 16, pp. 641-647, 1994.

[4] A. Mehnert and P. Jackway, "An improved seeded region growing algorithm,"Pattern Recognition Letters, vol. 18, pp. 1065-1071, 1997.

[5] J. Fan, G. Zeng, M. Body, and M.-S. Hacid, "Seeded region growing: an extensive and comparative study,"Pattern Recognition Letters, vol. 26, pp. 1139-1156, 2005

[6] F. Y. Shih and S. Cheng, "Automatic seeded region growing for color image segmentation,"Image and Vision Computing, vol. 23, pp. $877-886,2005$

[7] C. K. Gour, D. Laurence, and S. Mahbubur Rahaman, "Review of fuzzy image segmen-tation techniques," Design and Management of Multimedia Information Sys-tems: Opportunities and Challenges, I. G. I. (IGI), pp. 282-314, 2001

[8] J. C. Bezdek, J. Keller, R. Krisnapuram, and N. R. Pal, Fuzzy models and algorithms for pattern recognition and image processing vol. 4: Springer, 2005.

[9] J. V. de Oliveira and W. Pedrycz, Advances in Fuzzy Clustering and its Applications, Wiley Online Library, 2007.

[10] M. Hasanzadeh and S. Kasaei, "Multispectral brain MRI segmentation using genetic fuzzy systems," in Proc. ISSPA 2007. 9th International Symposium on Signal Processing and Its Applications, 2007, 2007, pp. $1-4$.

[11] G. C. Karmakar and L. S. Dooley, "A generic fuzzy rule based image segmentation algorithm,"Pattern Recognition Letters, vol. 23, pp. 1215-1227, 2002.

[12] K. S. Sundareswaran, D. H. Frakes, and A. P. Yoganathan, "Rule-based fuzzy vector median filters for 3D phase contrast MRI segmentation," Electronic Imaging 2008, pp. 68140F-68140F-14, 2008.

[13] C. Jawahar, P. K. Biswas, and A. Ray, "Analysis of fuzzy thresholding schemes,"Pattern Recognition, vol. 33, pp. 1339-1349, 2000.

[14] S. Pal, A. Ghosh, and B. U. Shankar, "Segmentation of remotely sensed images with fuzzy thresholding, and quantitative evaluation," International Journal of Remote Sensing, vol. 21, pp. 2269-2300, 2000.

[15] J. Kim, W. Cai, S. Eberl, and D. Feng, "Real-time volume rendering visualization of dual-modality PET/CT images with interactive fuzzy thresholding segmentation," IEEE Trans. on Information Technology in Biomedicine, vol. 11, pp. 161-169, 2007.

[16] J.-H. Xue, S. Ruan, B. Moretti, M. Revenu, D. Bloyet, and W. Philips, "Fuzzy modeling of knowledge for MRI brain structure segmentation," in Proc. 2000 International Conference on Image Processing, 2000, pp. 617-620.

[17] S. Ruan, B. Moretti, J. Fadili, and D. Bloyet, "Segmentation of magnetic resonance images using fuzzy Markov random fields," in Proc. 2001 International Conference on Image Processing, 2001, pp. 1051-1054.

[18] X. Lu, F. Zhou, and J. Zhou, "Synthetic aperture radar image segmentation based on improved fuzzy Markov random field model," in Proc. ISSCAA 2006. 1st International Symposium on Systems and Control in Aerospace and Astronautics, 2006, pp. 4-1208.

[19] M.-E. Algorri and F. Flores-Mangas, "Classification of anatomical structures in MR brain images using fuzzy parameters," IEEE Transactions on Biomedical Engineering, vol. 51, pp. 1599-1608, 2004.

[20] Z. Xiang, Z. Dazhi, T. Jinwen, and L. Jian, "A hybrid method for 3D segmentation of MRI brain images," in Proc. 2002 6th International Conference on Signal Processing, 2002, pp. 608-611.

[21] S. G. Dellepiane, F. Fontana, and G. L. Vernazza, "Nonlinear image labeling for multivalued segmentation," IEEE Transactions on Image Processing, vol. 5, pp. 429-446, 1996.

[22] J. K. Udupa and S. Samarasekera, "Fuzzy connectedness and object definition: theory, algorithms, and applications in image segmentation," Graphical Models and Image Processing, vol. 58, pp. 246-261, 1996.

[23] P. K. Saha and J. K. Udupa, "Relative fuzzy connectedness among multiple objects: theory, algorithms, and applications in image segmentation," Computer Vision and Image Understanding, vol. 82, pp. $42-56,2001$.

[24] Y. Zhuge, J. K. Udupa, and P. K. Saha, "Vectorial scale-based fuzzy-connected image segmentation," Computer Vision and Image Understanding, vol. 101, pp. 177-193, 2006.

[25] H. Suzuki and J.-I. Toriwaki, "Automatic segmentation of head MRI images by knowledge guided thresholding," Computerized Medical Imaging and Graphics, vol. 15, pp. 233-240, 1991.

[26] R. Pohle and K. D. Toennies, "Segmentation of medical images using adaptive region growing," in Proc. SPIE Medical Imaging, 2001, pp. 1337-1346.

[27] S. G. Dellepiane and F. Fontana, "Extraction of intensity connectedness for image processing," Pattern Recognition Letters, vol 16, pp. 313-324, 1995.

[28] Brainweb. (2012). Simulated Brain Database. [Online]. Available: www.bic.mni.mcgill.ca/brainweb

[29] I. I. B. S. Repository. (2012). Center for Morphometric Analysis at Massachusetts General Hospital. [Online]. Available: http://www.cma.mgh.harvard.edu/ibsr

[30] X.-Z. J. Luo, D. N. Kennedy, and Z. Cohen, "Neuroimaging informatics tools and resources clearinghouse (NITRC) resource announcement," Neuroinformatics, vol. 7, pp. 55-56, 2009.

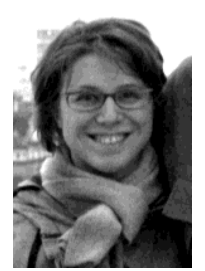

Sonia Nardotto was born in Albenga, Italy on November 18, 1987. She obtained the "Laurea" degree (BSc) in March 2010 and the "Laurea Magistrale" degree (MSc) in September 2012 both in Biomedical Engineering at University of Genoa. She developed her master thesis on "An automatic method for segmentation of multiparametric medical volumes".

Since November 2012 she has been cooperating with NUMIP area of the Signal \& Image Processing group at DITEN (Department of Naval Electrical Electronical and Telecommunication Engineering) in research activities concerning Image Processing and Data Fusion algorithms. Since January 2013 she is a Ph.D. student in Information and Knowledge Science and Technology: her research topics are Techniques of non-linear processing, segmentation, data fusion and analysis of the quality of digital images. She is co-author of paper presented at international conferences. She is also an IEEE Student Member associated to IEEE Signal Processing Society. 


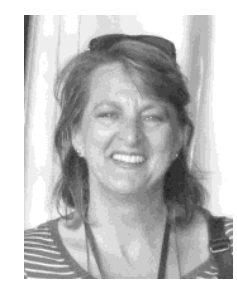

Silvana G. Dellepiane graduated in 1986 with honours. In 1990 she received the Ph.D. degree in Electronic Engineering and Computer Science. In 1992 she became a researcher (assistant professor) in the Department of Biophysical and Electronic Engineering (DIBE), University of Genoa, where she is an associated Professor in the ING_INF03 area. She has taught in Signal Theory and Pattern Recognition. At present, she is a professor of Electrical Communications, Statistical Methods, Signal and Image Processing and Recognition, in the courses of Telecommunications Engineering and Bioengineering. She is responsible for the NUMIP research area of the Signal Processing and Telecommunications (SP\&T) laboratory at DIBE.
Prof. Dellepiane has gained wide scientific and technical experience in multi-dimensional data processing. Her main research interests include the use of context and fuzzy systems for multi-dimensional data processing, segmentation, supervised methods for the processing of remote sensing SAR images, and non-linear adaptive processing of digital signals. Her application domains are mainly telemedicine and remote sensing.

Silvana Dellepiane is a reviewer for various journals. She was invited at some international conferences and schools for tutorials and lessons. She has participated, at the organization and scientific levels, in the research activities concerning various CNR, ASI, MIUR and UE projects. She is a member of the Technical Committee "Bio Imaging and Signal Processing" of IEEE Signal Processing Society. 